\title{
Iterative Fourier Transform Algorithm Based on the Segmentation of Target Image for a High-Speed Binary Spatial Light Modulator
}

\author{
Yeonsu Im ${ }^{1}$, Hwi Kim², and Joonku Hahn ${ }^{1}$ * \\ ${ }^{1}$ School of Electronics Engineering, Kyungpook National University, \\ 80 Daehak-ro, Buk-gu, Daegu 702-701, Korea \\ ${ }^{2}$ Department of Electronics and Information Engineering, College of Science and Technology, \\ Korea University, Sejong Campus, 2511 Sejong-ro, Sejong 339-700, Korea
}

(Received March 11, 2015 : revised March 30, 2015 : accepted March 30, 2015)

\begin{abstract}
A digital micro-mirror device (DMD) has the potential to modulate an incident wave with high speed, and the application for holographic display has been studied by many researchers. However, the quality of reconstructed image isn't good in comparison with that from a gray-scale amplitude-only hologram since it is a binary amplitude-only spatial light modulator (SLM). In this paper, we suggest a method generating a set of binary holograms to improve the quality of the reconstructed image. Here, we are concerned with the case for which the object plane is positioned at the Fourier domain of the plane of the SLM. In this case, any point in the Fourier plane is related to all points in the hologram. So there is a chance to generate a set of binary holograms illuminated by incident wave with constant optical power. Moreover, we find an interesting fact that the quality of reconstructed image is improved when the spatial frequency bandwidth of the binary hologram is limited. Therefore, we propose an iterative segmentation algorithm generating a set of binary holograms that are designed to be illuminated by the wave with constant optical power. The feasibility of our method is experimentally confirmed with a DMD.
\end{abstract}

Keywords: Digital holography, Spatial light modulators, Iterative Fourier transform algorithm

OCIS codes : (090.1995) Digital holography; (230.6120) Spatial light modulators; (090.2870) Holographic display

\section{INTRODUCTION}

Electronic holographic displays require a large number of data points, and a digital micro-mirror device (DMD) has a benefit to be applied for electronic holographic displays due to the modulation speed. In the DMD, each pixel has two stable states, and it can be turned on and off mechanically. Therefore, this is a binary amplitude-only spatial light modulator (SLM) and it is natural that the quality of reconstructed image is worse than that of a gray-scale amplitude-only SLM.

Early research on binary holograms was performed by Brown and Lohmann [1-3]. In these studies, there was some freedom to design the structure of every pixel independently where each pixel presents both amplitude and phase modulations of the optical wave. However, the shape of all pixels in the $\mathrm{DMD}$ are the same and there is no room to present complex modulation. But binary amplitude-only modulation has an interesting property. It represents +1 and 0 and it can be understood as a bias-shifted binary phase-only modulation that represents +0.5 and -0.5 . In this case, the bias is the DC-term in the Fourier domain. This conversion is an analogy to the optical single side-band filter usually used in electronic holography [4].

There have been remarkable studies to represent a gray-scale amplitude-only hologram [5-7] or a complex hologram [8] by using the DMD. Takaki suggested methods to display a single gray-scale hologram by using a set of several binary holograms. Here, the holograms are sequentially presented with different intensities of the incident wave. On the other hand, Onural proposed a complex hologram using two binary holograms. One is responsible for the real part and the other is responsible for the imaginary part. In these previous studies, the hologram is decomposed at the SLM plane. However, there is a new way to decompose the hologram in the Fourier

\footnotetext{
*Corresponding author: jhahn@knu.ac.kr

Color versions of one or more of the figures in this paper are available online.
} 
domain instead of in the SLM plane. In this paper, we are concerned with the case for which the object plane is positioned at the Fourier domain. In this situation, there is a great advantage for keeping the optical power of incident wave constant. It results from the fact that any point in the Fourier plane is related to all points in the hologram.

In this paper, we propose a new algorithm to decompose a grayscale hologram in the Fourier domain. We find an interesting property of a binary hologram that the quality of the reconstruction image is improved when the spatial frequency bandwidth of the hologram is severely limited. The iterative method has great advantages to increase the diffraction efficiency and regularity of the reconstructed image $[9,10]$. In this paper, an iterative segmentation algorithm is applied to generate a set of binary holograms where the segmentation of each hologram is carefully defined. Last, we demonstrate our decomposition method experimentally to prove its feasibility.

\section{BAND-LIMITATION OF BINARY HOLOGRAM}

In digital holography, Fourier optics is very useful for optical signal processing. In this paper, we are concerned with the Fourier optic situation where the decomposition is based on the segmentation in the Fourier plane. Figure 1 shows the object wave and the twin wave positioned at Fourier optics. The input plane $(x, y)$ is mapped to the output plane $(\xi, \eta)$ by the Fourier transform whose relation is represented by

$$
U(x, y)=\int_{-\infty}^{\infty} \int_{-\infty}^{\infty} U(\xi, \eta) \exp \left[-j 2 \pi\left(\xi \frac{x}{\lambda f}+\eta \frac{y}{\lambda f}\right)\right] d \xi d \eta
$$

Here, $U(\xi, \eta)$ is the wave modulated by the hologram. When an amplitude-only SLM is applied the twin wave appears

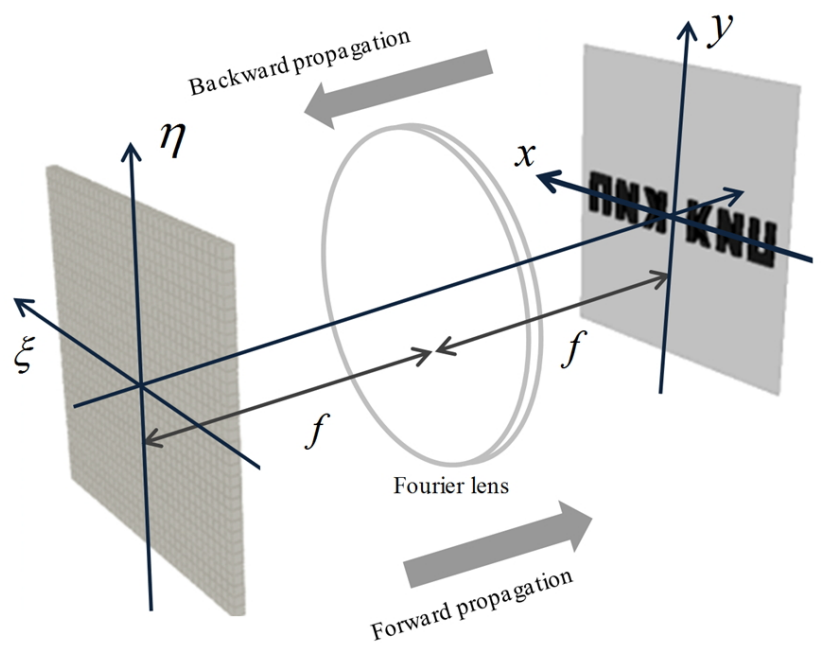

FIG. 1. Object wave and twin wave at Fourier optics. symmetrical to the object wave at the Fourier plane since the twin wave is the conjugate of the object wave. Therefore, the sign of the phase of the twin wave is different from that of the object wave at two points which are symmetric to each other around the origin.

The DMD is also an amplitude-only SLM and it inevitably reconstructs both the object wave and the twin wave simultaneously. The Fourier plane is the best position to separate both of them. For this purpose, the spatial frequency of the object wave needs to be limited within the single-side bandwidth. Figure 2(a) shows a band-limited target image. In this paper, the hologram is computed by iterative Fourier transform algorithm(IFTA). This IFTA is well-known as the algorithm generating the phase-only hologram. In this algorithm, the Fourier optics is proper for generation of the phase-only hologram since the Fourier transform makes any point in the Fourier plane related to all points in the hologram.

There is an obvious reason to apply this IFTA for our purpose. Even though the DMD modulates the amplitude of the wave, it is only binary control. Therefore, when the complex hologram is encoded into the DMD, most of the continuous amplitude information is lost. As previously explained, binary amplitude-only modulation can be converted into the binary phase-only modulation by biasing the DC term in the complex plane. The phase information is significantly different from the amplitude information since the phase is wrapped within $2 \pi$, whereas there is no upper limit in the amplitude information. The binarization of the amplitude-only hologram is severely affected by the criteria which determines whether a point is 0 or 1 . On the other hand, the binarization of the phase-only hologram has an advantage that the quality of the reconstructed image is relatively robust in the criteria. A binary hologram from the phase-only hologram and its numerical reconstruction are shown in Fig. 2(b) and(c)

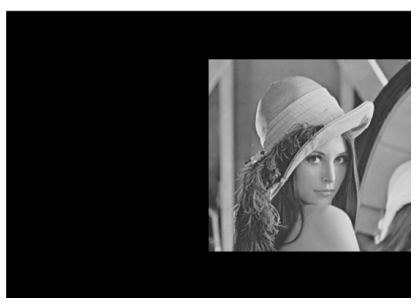

(a)

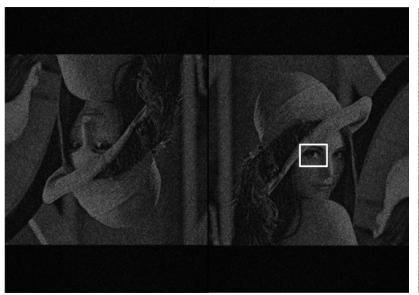

(c)

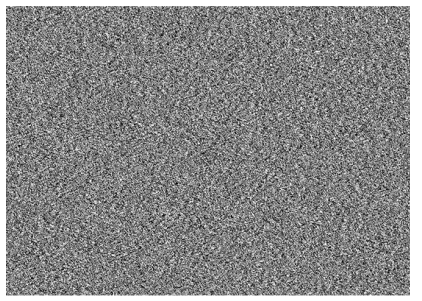

(b)

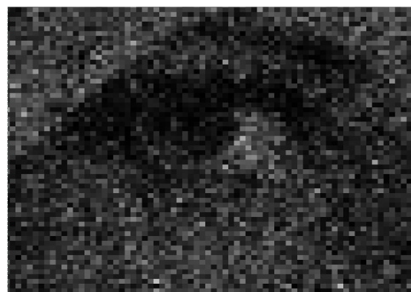

(d)
FIG. 2. Numerical reconstruction from a single binary hologram. (a) Band-limited target image. (b) Binary hologram and (c) reconstruction image. (d) Enlarged image of rectangular area in Fig. 2(c). 


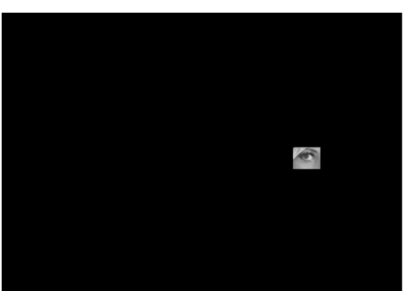

(a)

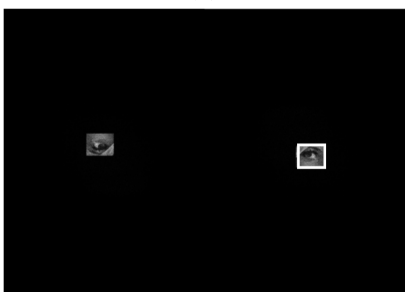

(c)

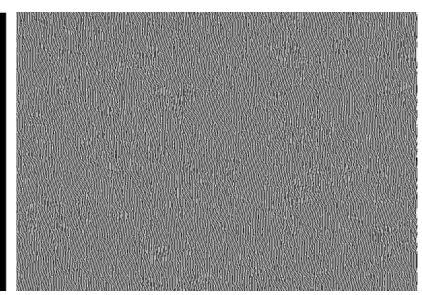

(b)

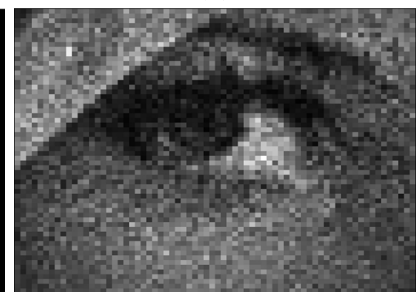

(d)
FIG. 3. Numerical reconstruction from a single binary hologram. (a) Narrowly band-limited target image. (b) Binary hologram and (c) reconstruction image. (d) Enlarged image of rectangular area in Fig. 3(c).

respectively. It is clear that the twin image appears in the other side and the quality of the reconstruction image becomes bad when the hologram is binarized.

For improvement of the image quality, the band-limitation of the binary hologram in the spatial frequency domain is very effective. Figure 3 shows the case that a target image is only a small portion of the object in the spatial frequency domain. The binary hologram is obtained from the phase-only hologram as shown in Fig. 3(b). The quality of the reconstructed image in Fig. 3(d) is much better than that of the result in Fig. 2(d).

This result is reasonable from the viewpoint of the information theory. The IFTA generates phase-only holograms and the amount of the information decreases abruptly when the holograms are binarized, For example, an 8 bit gray-scale image has 128 times more information than that of a binary image. When the bandwidth of the spatial frequency is limited, relatively many pixels are used for reconstructing a point in the spatial frequency domain. Also, the band-limitation means that only a small region in spatial frequency is concerned for reconstruction of the binary hologram and the interference pattern obviously appears in Fig. 3.

\section{SEGMENTATION OF TARGET IMAGE}

By limiting the spatial-frequency bandwidth of the hologram, the quality of the reconstructed image is improved. Based on this assumption, we propose the way that the target image is divided into several segments and a set of binary holograms for each of the segments is generated based on IFTA. In previous studies [5-8], the optical power of a light source should be differently controlled according to the sub-holograms. In our method, the size of the segments is determined in

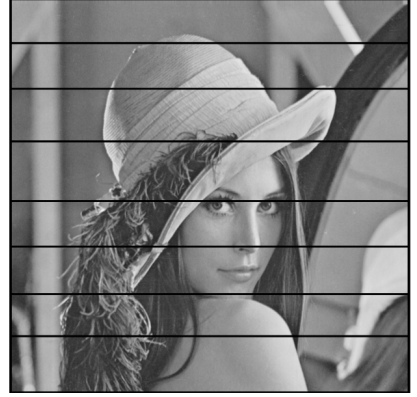

(a)

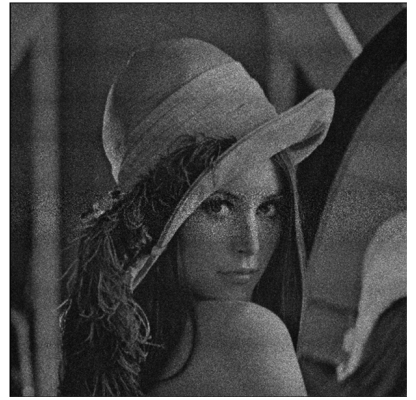

(b)
FIG. 4. (a) Segmentation of target image and (b) numerical reconstruction of binary holograms corresponding to each segmentation.

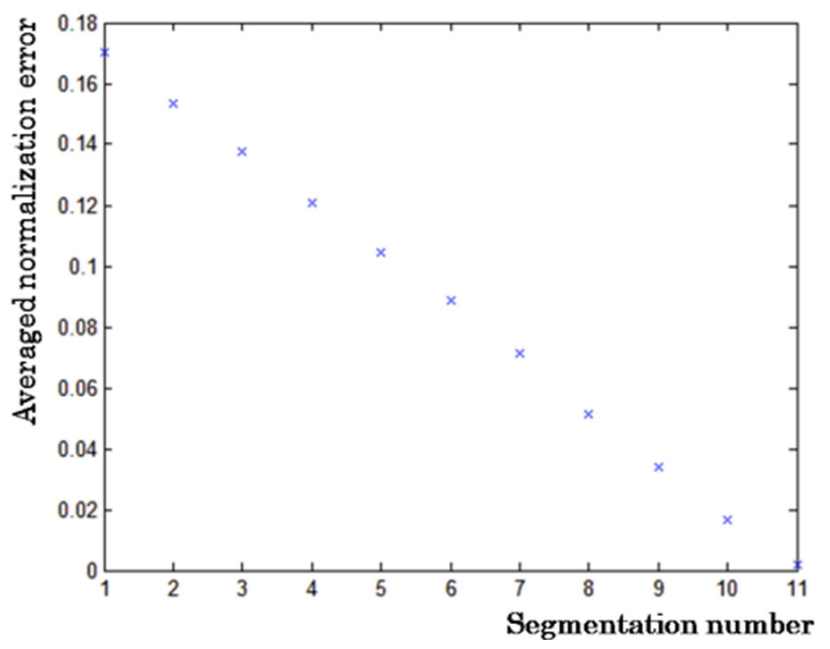

FIG. 5. Numerical error from a single binary hologram with segmentation number.

order that the total intensity of each segment becomes the same. This results in a great advantage that the intensity of incident wave can remain constant. Figure 4 shows the simulation results when the target is vertically divided into eight segments. In Fig. 4(b), only the object part is cropped for simplicity and the resultant reconstructed image looks better than the result from a single hologram in Fig. 2(c).

In order to check the effect of the number of the segments, an averaged normalized error is defined by

$$
E_{a v}=\frac{\sum_{x} \sum_{y}\left|I_{r}(x, y)-I_{t}(x, y)\right| / I_{t}(x, y)}{\sum_{x} \sum_{y} 1} .
$$

Here, $I_{r}(x, y)$ is the intensity of the reconstructed wave and $I_{t}(x, y)$ is the intensity of the target image. The averaged normalized error decreases as the number of the segments increases as shown in Fig. 5. Even though the DMD can be operated in high speed, increase of the number of segments results in the increase of the operation time to display a 
whole set of binary holograms. Therefore, eight is a practical number of segments for the DMD to be used for electronic holographic display.

When the target is vertically segmented, the normalized error becomes significantly visible at the boundaries as shown in Fig. 4(b). To avoid this problem, we propose another way to assign the points of target image into sub-holograms. Figure 6 shows a map to assign each point by using a random distribution function. It is helpful to balance the total intensities in charge of each sub-hologram. As previously explained, the total intensities of the sub-hologram are designed to be the same in order to keep the illumination power constant. Therefore, when the target image is segmented, the intensity of each segment needs to be calculated. But when the pixels of the target image are randomly assigned to the sub-segments, the intensity of each segment becomes almost the same.

Figure 7 shows the numerical reconstructions from a single binary hologram and from a set of eight binary holograms

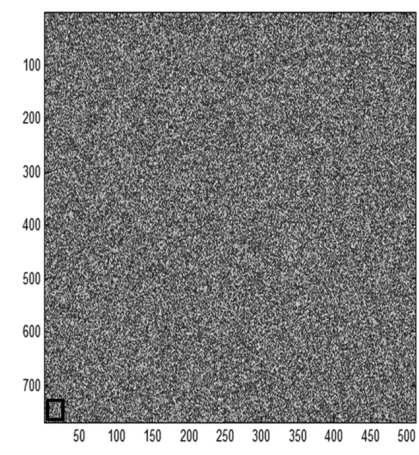

(a)

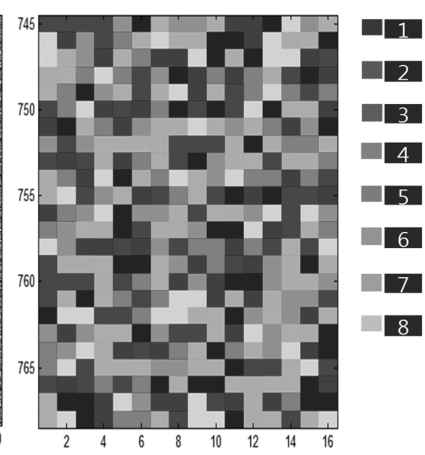

(b)
FIG. 6. (a) Segmentation according to random distribution function and (b) enlarged images of rectangular area in Fig. 6(a).

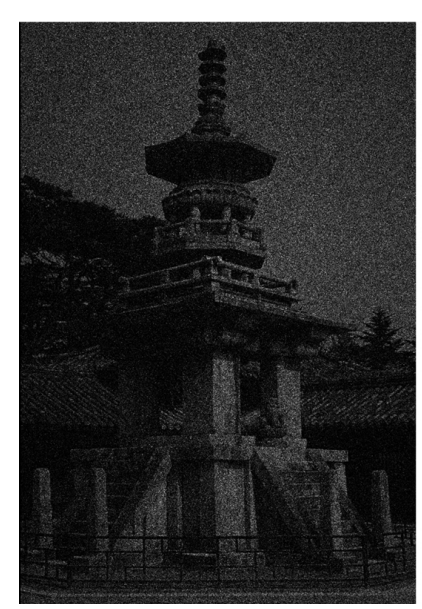

(a)

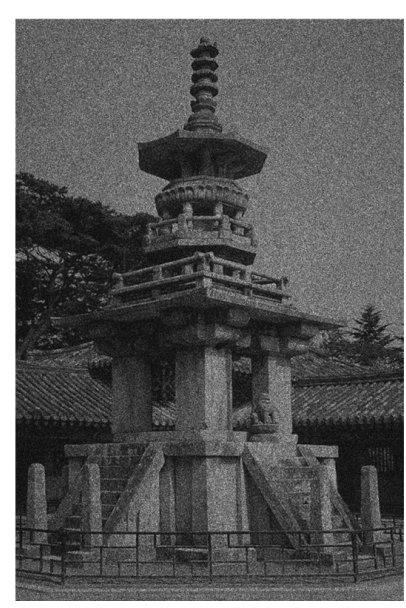

(b)
FIG. 7. Numerical reconstructions (a) from a single binary hologram and (b) from a set of eight binary holograms generated by segmentation algorithm. generated by our segmentation algorithm. Here, the target image is segmented by using the random function. The result from our method presents more details and higher contrast. For example, the fence around the pagoda and traditional roof behind it in Fig. 7(b) look much clearer than those in Fig. 7(a).

To compare the qualities of these results numerically, we introduce a mean square error (MSE) which is defined by

$$
M S E=\sqrt{\frac{\sum_{x} \sum_{y}\left[\left|I_{r}(x, y)-I_{t}(x, y)\right| / I_{t}(x, y)\right]^{2}}{\sum_{x} \sum_{y} 1}} .
$$

The MSE is expected to represent the irregularity of reconstructed image since it has similar form to the standard deviation. When the image is reconstructed from a single binary hologram, the averaged normalized error is 0.6344 and the MSE is 0.3221. On the other hand, when the image is reconstructed from a set of eight binary holograms, the averaged normalized error becomes 0.5312 and the MSE is 0.2962 . We think that this relatively small improvement results from the segmentation using a random distribution function. Even though this random distribution function is used to diminish the distinct error along the boundaries among vertically divided segmentations, ironically the length of boundary of segmentation from the random distribution function is much longer than that of the simply divided segmentation.

\section{EXPERIMENTAL RESULTS}

Figure 8 shows the layout of the optical system to demonstrate the feasibility of our decomposition method. A doublet lens with diameter of $50 \mathrm{~mm}$ and focal length of $150 \mathrm{~mm}$ is used as Fourier lens and $4 f$ system is inserted between the DMD and the Fourier lens. $4 f$ system is useful to place the optical mask to filter out the twin wave and higher-order diffraction terms. In this paper, the optical mask is not inserted

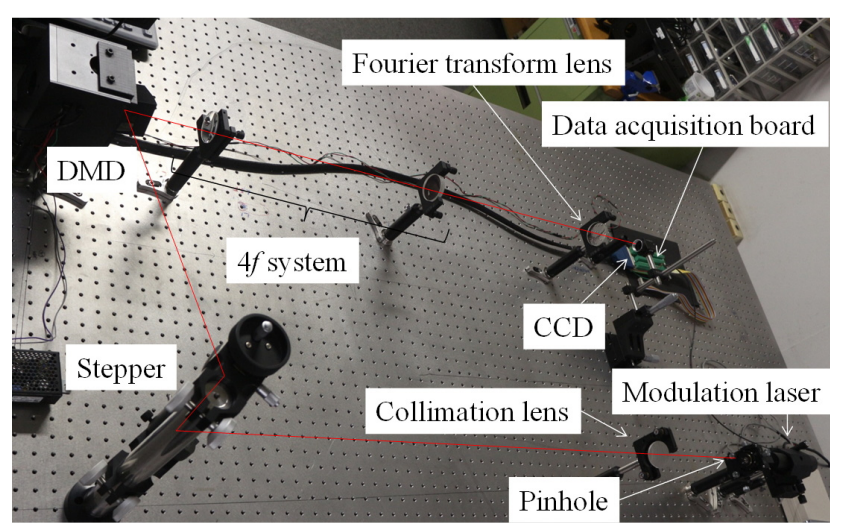

FIG. 8. Experimental setup of sequential display of a set of binary hologram using the DMD. 


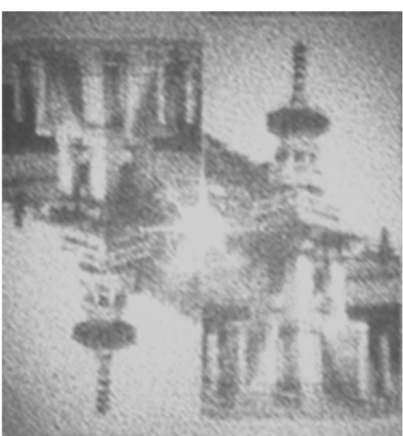

(a)

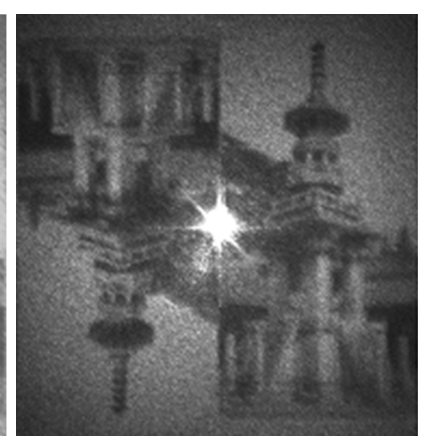

(b)
FIG. 9. Optical reconstruction images (a) from a single binary hologram and (b) from binary holograms generated by segmentation algorithm.

to check the quality of reconstruction image without any conflicts. A diode laser with wavelength of $635 \mathrm{~nm}$ is applied and the optical wave is collimated with pinhole and collimation lens. The DMD with XGA resolution is used for binary modulation. The cage of the DMD is designed for a cube beamsplitter to be attached on it. By this beamsplitter, the collimated wave enters obliquely at 24 degrees with respect to the normal direction of the DMD. The modulated wave passes through the beamsplitter again. Then it passes the $4 f$ system and the Fourier lens. Finally, the reconstruction image is measured by the imaging sensor. Here, the CCD, FL2-14S3M-C manufactured by Pointgrey is used as the imaging sensor.

Figure 9 shows optical reconstruction images from a single binary hologram and from binary holograms generated by our segmentation algorithm. The result from our method has more visibility in detail and higher contrast as expected. Unfortunately, it is not easy to compute the averaged normalized error and the MSE from the captured images. First, the sampling interval of the CCD is different from the interval of the spatial frequency of the hologram by discrete Fourier transform. Second, it is not easy to fairly compare the reconstructed image from a single binary hologram with the reconstructed image from eight binary hologram since the latter is a time-averaged image. It means that the exposure time of the $\mathrm{CCD}$ is very critical in the experiment. Therefore, the images in Fig. 9 are a little blurred due to the long exposure time.

\section{CONCLUSION}

Recently, the DMD has received large interest due to its ability to modulate the optical wave by high speed. However, it has an intrinsic disadvantage in the quality of the reconstructed image since it is a binary amplitude-only SLM. To overcome this problem, some methods have been previously suggested where a complex hologram is decomposed into several binary holograms in the hologram plane. In this paper, we propose a new decomposition method based on the segmentation of the hologram in the spatial frequency domain. This has significant benefits that the optical power of illumination wave doesn't have to be changed. The phase-only hologram for each segment is computed by IFTA and binarized. Generally, when the amplitude-only hologram is binarized, the quality is very sensitive to the binarization criteria. However, our method is very robust in binarization criteria since the phase information is already wrapped. We confirmed the feasibility of our method experimentally.

\section{ACKNOWLEDGMENT}

This research was financially supported by the Ministry of Education (MOE) and National Research Foundation of Korea (NRF) through the Human Resource Training Project for Regional Innovation (NRF-2012H1B8A2026187).

\section{REFERENCES}

1. B. R. Brown and A. W. Lohmann, "Complex spatial filtering with binary masks," Appl. Opt. 5, 967-969 (1966).

2. A. W. Lohmann and D. P. Paris, "Binary Fraunhofer holograms, generated by computer," Appl. Opt. 6, 1739-1748 (1967).

3. B. R. Brown and A. W. Lohmann, "Computer-generated binary hologram,” IBM J. Res. Dev. 13, 160-168 (1969).

4. Y. Takaki and Y. Tanemoto, "Band-limited zone plates for single-sideband holography," Appl. Opt. 48, H64-H70 (2009).

5. Y. Takaki, M. Yokouchi, and N. Okada, "Improvement of grayscale representation of the horizontally scanning holographic display," Opt. Express 18, 24926-24936 (2010).

6. Y. Takaki and M. Yokouchi, "Speckle-free and grayscale hologram reconstruction using time-multiplexing technique," Opt. Express 19, 7567-7579 (2011).

7. T. Kurihara and Y. Takaki, "Speckle-free, shaded 3D images produced by computer-generated holography," Opt. Express 21, 4044-4054 (2013).

8. E. Ulusoy, L. Onural, and H. M. Ozaktas, "Full-complex amplitude modulation with binary spatial light modulators," J. Opt. Soc. Am. A 28, 2310-2321 (2011).

9. H. Kim, B. Yang, and B. Lee, "Iterative Fourier transform algorithm with regularization for the optimal design of diffractive optical elements," J. Opt. Soc. Am. A 21, 2353-2365 (2004).

10. H. Kim and B. Lee, "Diffractive optical element with apodized aperture for shaping vortex-free diffraction image," Jpn. J. Appl. Phys. 43, 1530-1533 (2004). 